\title{
IFN- $\alpha$ inhibits HBV transcription and replication in cell culture and in humanized mice by targeting the epigenetic regulation of the nuclear cccDNA minichromosome
}

\begin{abstract}
Laura Belloni, 1,2,3 Lena Allweiss, ${ }^{4}$ Francesca Guerrieri, 1,3,5 Natalia Pediconi, ${ }^{1,3,5}$ Tassilo Volz, ${ }^{4}$ Teresa Pollicino, ${ }^{6}$ Joerg Petersen,7 Giovanni Raimondo, 6 Maura Dandri,4 and Massimo Levrero ${ }^{1,2,3,5}$

${ }^{1}$ EAL Inserm U785, Sapienza University of Rome, Rome, Italy. ²Laboratory of Gene Expression, Fondazione A. Cesalpino, Rome, Italy. ${ }^{3}$ Department of Internal Medicine, University La Sapienza, Rome, Italy. ${ }^{4}$ Department of Internal Medicine, University Medical Hospital Hamburg-Eppendorf, Hamburg, Germany. ${ }^{5}$ Rome Oncogenomic Center, Istituto Regina Elena, Rome, Italy. ${ }^{6}$ Department of Internal Medicine, University of Messina, Messina, Italy. IIFI Institute for Interdisciplinary Medicine, Asklepios Clinic St. Georg, Hamburg, Germany.
\end{abstract}

\begin{abstract}
HBV infection remains a leading cause of death worldwide. IFN- $\alpha$ inhibits viral replication in vitro and in vivo, and pegylated IFN- $\alpha$ is a commonly administered treatment for individuals infected with HBV. The HBV genome contains a typical IFN-stimulated response element (ISRE), but the molecular mechanisms by which IFN- $\alpha$ suppresses HBV replication have not been established in relevant experimental systems. Here, we show that IFN- $\alpha$ inhibits HBV replication by decreasing the transcription of pregenomic RNA (pgRNA) and subgenomic RNA from the HBV covalently closed circular DNA (cccDNA) minichromosome, both in cultured cells in which HBV is replicating and in mice whose livers have been repopulated with human hepatocytes and infected with HBV. Administration of IFN- $\alpha$ resulted in cccDNA-bound histone hypoacetylation as well as active recruitment to the cccDNA of transcriptional corepressors. IFN- $\alpha$ treatment also reduced binding of the STAT1 and STAT2 transcription factors to active cccDNA. The inhibitory activity of IFN- $\alpha$ was linked to the IRSE, as IRSE-mutant HBV transcribed less pgRNA and could not be repressed by IFN- $\alpha$ treatment. Our results identify a molecular mechanism whereby IFN- $\alpha$ mediates epigenetic repression of HBV cccDNA transcriptional activity, which may assist in the development of novel effective therapeutics.
\end{abstract}

\section{Introduction}

Hepatitis B Virus (HBV) infection remains a major health problem worldwide despite the availability of a highly effective preventive vaccine. $\mathrm{HBV}$ is a noncytopathic hepatotropic DNA virus that belongs to the family Hepadnaviridae, whose members share a distinctive strategy for replication. HBV replication occurs in the cytoplasm within viral capsids (core particles), where a genomesized RNA replicative intermediate, termed the pregenome (pgRNA), is converted by the virally encoded RNA-dependent and DNA-dependent reverse transcriptase/polymerase into a specific open circular (OC) duplex DNA (1). Transcription in the nucleus of the pgRNA from the covalently closed circular DNA (cccDNA) is the critical step for genome amplification and ultimately determines the rate of HBV replication (2). The cccDNA, which also serves as the template for the transcription of all viral messenger RNAs, is organized into a minichromosome in the nuclei of infected hepatocytes by histone and nonhistone proteins, and its function is regulated, similarly to cellular chromatin, by the activity of nuclear transcription factors, transcriptional coactivators and corepressors, and chromatin-modifying enzymes (2-4).

Current antiviral therapies involve the use of nucleoside analogs and pegylated IFN- $\alpha$ (5). IFN- $\alpha$, a type I IFN, engages the IFN- $\alpha / \beta$ receptor complex to activate the intracellular Jak/Stat signaling pathway, which modulates the transcription of a diverse set of target genes, referred to as IFN-stimulated genes (ISGs) (6). ISG

Conflict of interest: The authors have declared that no conflict of interest exists. Citation for this article: J Clin Invest. 2012;122(2):529-537. doi:10.1172/JCI58847. modulation results in an antiviral response in target cells aimed at limiting both viral replication and spreading. IFN- $\alpha$ has been reported to inhibit HBV replication through a variety of mechanisms, including a block of RNA-containing core particle formation, an accelerated decay of replication-competent core particles, and degradation of the pgRNA (7-9).

An IFN-stimulated response element (ISRE) has been identified in the enhancer $1 / \mathrm{X}$ gene promoter region of the $\mathrm{HBV}$ genome (10), and IFN- $\alpha$ has been shown to suppress viral gene expression $(11,12)$. Subsequent studies failed to establish the role of IFN- $\alpha$, the HBV ISRE, and the STAT proteins on HBV transcription (13). However, most studies were conducted in vitro or in a nonreplicative context. We sought to employ relevant in vitro replicative models and in vivo infection systems to investigate whether IFN$\alpha$ targets cccDNA transcription to inhibit viral replication and attempted to define the molecular mechanisms of IFN- $\alpha$ repression. To this aim, we made use first of a plasmid-free HBV transfection cell-based replication assay relying on the generation of transcriptionally active nuclear cccDNA to replicate $\operatorname{HBV}(3,14)$. Second, SCID/beige mice transgenic for the urokinase plasminogen activator (uPA) under control of the albumin promoter were used to repopulate mouse livers with human hepatocytes derived from a single liver donor $(15,16)$. This model minimizes the impact of host variation factors and allows the investigation of in vivo interactions occurring between $\mathrm{HBV}$ and human hepatocytes, the natural target cell of infection and replication. Our results indicate that IFN- $\alpha$ suppresses HBV replication by targeting the epigenetic control of cccDNA function and transcription. 

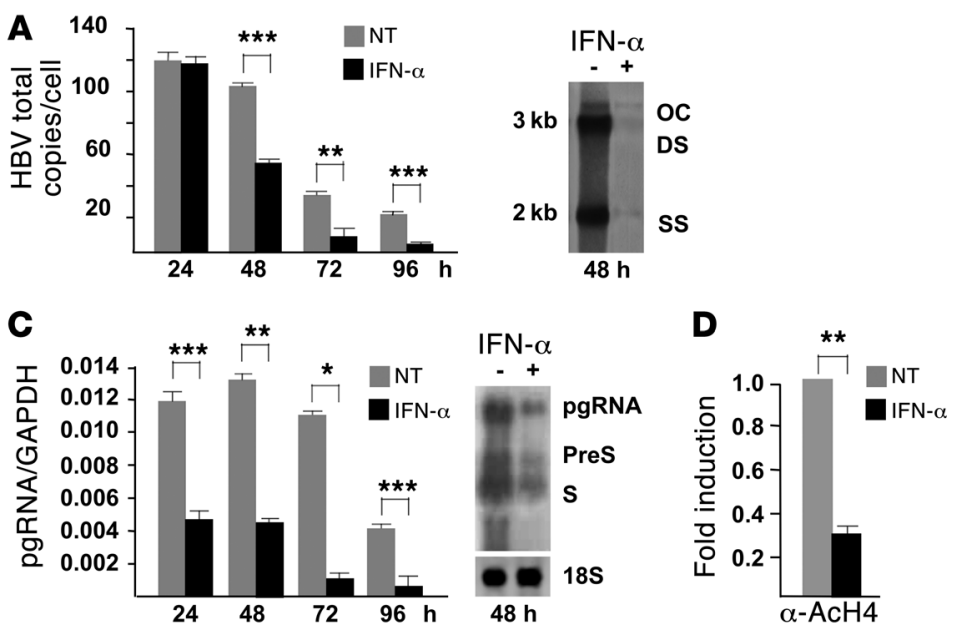
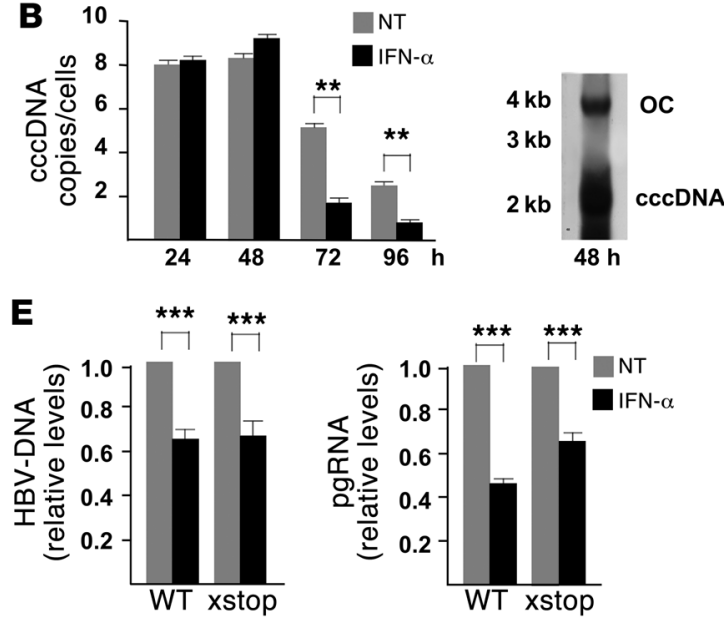

\section{Figure 1}

IFN- $\alpha$ inhibits HBV replication and cccDNA transcription in HCC cells. (A) Left panel: HepG2 cells were transfected with monomeric linear fulllength WT HBV adw (genotype A) genomes. HBV core particles were isolated from untreated and IFN- $\alpha$-treated cells at the indicated time points after transfection. Results are expressed as number of HBV DNA copies per transfected cell. Right panel: Southern blot hybridization. OC, open circular duplex HBV DNA; DS, double-stranded HBV DNA replicative intermediates; SS, single-stranded HBV DNA replicative intermediates. (B) Left panel: cccDNA levels (copies per transfected cell) were analyzed by qPCR with selective cccDNA primers and $\beta$-globin primers (DNA sample normalization). Right panel: Southern blot analysis. (C) Left panel: pgRNA levels were analyzed by qPCR using pgRNA selective primers and GAPDH primers (equal loading of each RNA sample). Right panel: Northern blot analysis. pgRNA, HBV pregenomic RNA. (D) Crosslinked chromatin was immunoprecipitated with a relevant control IgG or specific anti-AcH4 antibody and analyzed by qPCR with HBV cccDNA selective primers. Results are expressed as fold induction relative to the untreated cells using the comparative Ct method. (E) HepG2 cells were transfected with monomeric linear full-length WT or HBx mutant HBV genomes (4). Core particles HBV-DNA (left panel) and pgRNA (right panel) results are expressed as in Figure 1, A and C, respectively. All histograms show mean values from 3 independent experiments; bars indicate SD. $P$ values were determined using Student's $t$ test. ${ }^{*} P<0.05,{ }^{* *} P<0.01,{ }^{* * *} P<0.001$.

\section{Results}

IFN- $\alpha$ inhibits cccDNA transcription and HBV replication in HCC cells. Class I IFNs inhibit HBV replication in a variety of plasmidbased replication assays in HCC cell lines and nonhuman primary hepatocytes $(7,17-20)$. We examined the impact of IFN- $\alpha$ treatment on cccDNA transcription and HBV replication in a cccDNA-driven replication system $(3,4)$. Equivalent numbers of HepG2 cells were transfected with linear WT HBV (genotype A) genomes and exposed to IFN- $\alpha(1000 \mathrm{U} / \mathrm{ml})$. We confirmed that in IFN- $\alpha$-treated HepG 2 cells, STAT1 and STAT2 are rapidly phosphorylated (Supplemental Figure 1; supplemental material available online with this article; doi:10.1172/JCI58847DS1) and translocate to the nucleus to bind the promoter regions of ISGs to stimulate their transcription (data not shown and Supplemental Figure 2). Cells were harvested at the indicated times after transfection (Figure 1, A and B), cytoplasmic viral core particles were isolated, and capsid-associated HBV DNA was quantified by realtime PCR and analyzed by hybridization with a ${ }^{32}$ P-labeled fulllength HBV DNA probe. As shown in Figure 1, A and B, the level of capsid-associated HBV DNA and cccDNA molecules per cell was highest at 24 and 48 hours after transfection and decreased in the following days. IFN- $\alpha$ administration performed shortly after transfection strongly suppressed steady-state amounts of capsidassociated HBV DNA compared with the viral loads determined in untreated cells, although the number of cccDNA copies per cell was not modified by IFN- $\alpha$ at 24 and 48 hours of treatment (Figure 1B). Notably, Northern blot analysis revealed that IFN- $\alpha$ treatment also lowered the steady-state levels of HBV transcripts in HBV-replicating cells. Both $3.5 \mathrm{~kb}$ pgRNA and 2.4-2.1 mRNA spe- cies (preS/S RNA) levels were significantly reduced in IFN- $\alpha$-treated cells (Figure 1C). Quantification of pgRNA levels by real-time PCR showed a $60 \%$ to $70 \%$ decrease after 24 and 48 hours of IFN- $\alpha$ treatment (Figure 1D), without affecting levels of cccDNA copies per cell (Figure 1B). These results confirm that IFN- $\alpha$ inhibits HBV replication in our system and indicate that IFN- $\alpha$ lowers steadystate levels of HBV transcripts.

Several types of evidence support the notion that cccDNA transcription is controlled by epigenetic modifications of cccDNAbound histones. Indeed, HBV replication and cccDNA transcription are modulated by substances that interfere with the activity of known chromatin-modifying enzymes, such as class I/II histone deacetylases (3). Moreover, using the cccDNA ChIP assay, which couples a classical ChIP step with a cccDNA-specific real-time PCR to selectively detect the immunoprecipitation of cccDNA-bound histones and nonhistone proteins, we showed that cccDNA transcriptional activity and HBV replication are controlled, both in cell culture systems and in vivo in the liver of chronic HBV carriers, by the acetylation status of cccDNA-bound $\mathrm{H} 3$ and $\mathrm{H} 4$ histones $(2,3)$. We found that 48 hours after transfection, cccDNA-bound H4 histones were significantly hypoacetylated in IFN- $\alpha$-treated cells, thus linking the repression of HBV transcription by IFN- $\alpha$ with cccDNA-bound histone hypoacetylation (Figure 1D).

The regulatory protein $\mathrm{HBx}$ is recruited onto the cccDNA minichromosome in HBV-replicating cells (4) and is required for both cccDNA transcription and HBV replication in the context of a natural HBV infection (21). In the absence of HBx, cccDNAbound histones are hypoacetylated, and the cccDNA transcribes significantly less pgRNA (4). We sought to assess whether the 
A

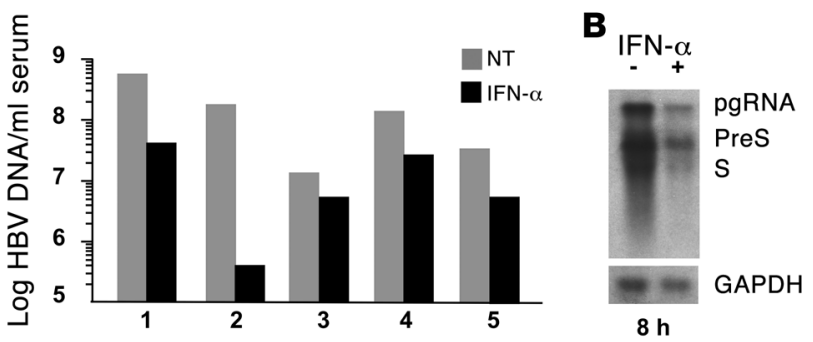

C
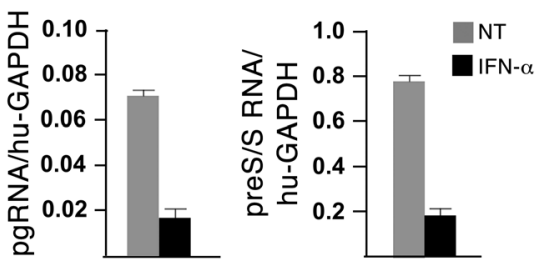

D

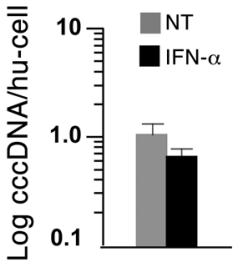

E

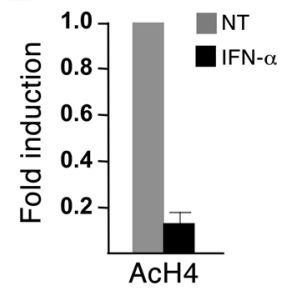

\section{Figure 2}

IFN- $\alpha$ inhibits cccDNA transcription and HBV replication in chimeric UPA/ SCID mice. Uninfected and chronically HBV infected (median, $2 \times 10^{8}$ serum HBV-DNA copies/ $\mathrm{ml}$ serum) chimeric UPA/SCID mice transplanted with thawed human hepatocytes received daily injections of human IFN- $\alpha$ (1,300 IU/g body weight) for 5 days or saline. (A) HBV viremia (log HBVDNA copies/ $\mathrm{ml}$ serum) were determined in individual animals $(n=5)$ at baseline, shortly before IFN treatment, and 8 hours after the last administration of IFN- $\alpha$. (B) Representative Northern blot analysis from one IFN- $\alpha-$ treated mouse and one untreated mouse. (C) Steady-state levels of pregenomic RNA (left panel) and preS/S RNAs (right panel). Intrahepatic preS/S RNAs were determined by subtracting pgRNA amounts from total HBV RNAs (pgRNA + preS/S RNA) estimated in the same RNA preparation as described in Methods, and values were normalized using humanspecific GAPDH primers. (D) Intrahepatic cccDNA loads in IFN- $\alpha$-treated $(n=5)$ and control (saline) HBV-infected mice $(n=5)$. Real-time qPCR analysis was performed using selective cccDNA primers, and $\beta$-globin primers were used to normalize cccDNA copies (median $+\mathrm{SD}$ ) per human hepatocyte (expressed as human genome equivalents) determined in chimeric livers. (E) Cross-linked chromatin from liver samples of chronically HBV-infected untreated and IFN- $\alpha$-treated (48 hours) UPA chimeric mice was immunoprecipitated with a relevant control IgG or specific anti-AcH4 antibody. Immunoprecipitated chromatin was analyzed by qPCR as in Figure 1D. All histograms show mean values from 3 independent experiments; bars indicate SD. repression of cccDNA transcriptional activity by IFN- $\alpha$ is mediated by or requires HBx. As shown in Figure 1E, IFN- $\alpha$ inhibited capsid-associated HBV DNA and pgRNA levels to a similar extent in HepG2 cells replicating WT or HBx mutant viruses, indicating that the repression of cccDNA transcriptional activity by IFN- $\alpha$ is $\mathrm{HBx}$ independent.

IFN- $\alpha$ inbibits cccDNA transcription and $H B V$ replication in chimeric uPA/SCID mice. The lack of efficient HBV infection models has greatly limited the study of the antiviral mechanisms induced by IFN- $\alpha$ in HBV-infected hepatocytes in vivo. To determine the impact of IFN treatment on intrahepatic viral activity, chimeric uPA/SCID mice were repopulated with primary human hepatocytes and infected with HBV in vivo (15). $10 \mathrm{HBV}$-infected mice displaying stable median viremia levels of $2 \times 10^{8} \mathrm{HBV}$ DNA copies $/ \mathrm{ml}$ (range, $2 \times 10^{7}$ to $6 \times 10^{8}$ ) received either daily injections of human IFN- $\alpha(n=5)$ or saline $(n=5)$. Comparative analysis of viremia changes performed in single mice after 5 days of IFN treatment showed a significant reduction in viremia (range, 0.4 to 2.5 log; median, $0.8 \mathrm{log}$ ) compared with baseline in all animals analyzed (Figure 2A). Similarly to what we observed in HBV-replicating HCC cell lines, IFN- $\alpha$ treatment reduced both the levels of pgRNA and subgenomic RNAs (preS/S RNA) in all animals analyzed, as shown by Northern blot (Figure 2B) and real-time RT-PCR analysis (Figure 2C) performed on liver tissues obtained 8 hours after the last IFN application, whereas no changes in the intrahepatic cccDNA load were observed (Figure 2D). Notably, the reduction of HBV RNA transcripts was accompanied by a sharp reduction of cccDNAbound histone $\mathrm{H} 4$ acetylation (Figure $2 \mathrm{E}$ ). These data indicate that IFN- $\alpha$ exhibits a direct antiviral effect in infected primary human hepatocytes by inducing the hypoacetylation of cccDNA-bound histones and decreasing the levels of HBV transcripts in vivo without any measurable effect on the size of the nuclear cccDNA pool.
The HBV ISRE mediates STAT1 and STAT2 recruitment on the cccD$N A$ and IFN- $\alpha$ transcriptional repression. Next, we sought to investigate whether the ISRE element present on the HBV cccDNA is functional in vivo and might be therefore involved in the HBV transcriptional repression observed after IFN- $\alpha$ treatment. As shown in Figure 3A, STAT1, STAT2, and their phosphorylated forms all bind to the cccDNA in unstimulated cells. After IFN- $\alpha$ treatment, both cccDNA-bound phospho-STAT1 and phosphoSTAT2 and, to a lesser extent, STAT1 and STAT2, recruitment, are greatly reduced (Figure $3 \mathrm{~B}$ ). These results indicate that the HBV ISRE is functional in the context of HBV replication and that STAT binding to the cccDNA changes upon IFN- $\alpha$ treatment. Although we do not have formal evidence, these results also suggest that, in response to IFN- $\alpha$, there is a dynamic change in the quality of the complexes bound to the HBV ISRE with loss of phosphorylated STAT2 and STAT1 and a decrease of unphosphorylated STAT proteins. In this respect, it is noteworthy that chronic HBV infection of human hepatocytes transplanted in uPA/SCID mice impairs the induction of IFN- $\alpha$ target genes by inhibiting nuclear translocation of STAT1 (16). The presence of STAT 1 and STAT 2 complexes on the cccDNA before IFN- $\alpha$ treatment apparently defies the classical paradigm of the STATs as latent transcription factors in the cytoplasm, entering the nucleus to induce gene expression only in response to cytokine stimulation to bind DNA and activate their transcriptional program, but it is not unprecedented. Using a ChIP-Seq approach, it has been shown that more than $85 \%$ of STAT1-binding peaks overlap before and after IFN- $\gamma$ stimulation (22). Recently, using a ChIP-on-chip approach on a large subset of IFN- $\alpha$ direct target genes, we could show an occupancy of ISRE sites by STAT2 in about half of the promoters we studied in unstimulated HCC cells lines and primary human hepatocytes (23). 
A

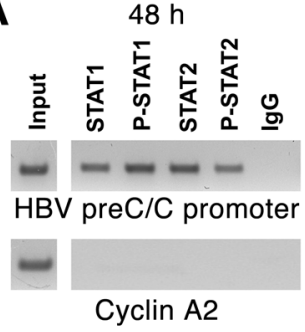

B

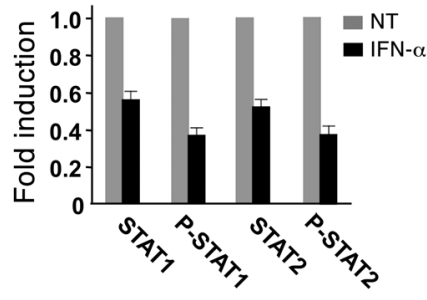

Figure 3

STAT1 and STAT2 transcription factors are recruited on the cccDNA (A) Cross-linked chromatin from HepG2 cells transfected with monomeric linear full-length HBV DNA was immunoprecipitated with a relevant control IgG or specific anti-STAT1, anti-phospho-STAT1, antiSTAT2, and anti-phospho-STAT2 antibodies. Immunoprecipitated chromatin samples were analyzed by real-time PCR with either HBV cccDNA selective primers (upper panel) or primers specific for the cyclin A2 coding region as a negative control (lower panel). (B) Chromatin was prepared from untreated and IFN- $\alpha$-treated HepG2 cells transfected with WT HBV genomes. Immunoprecipitated chromatin was analyzed and results expressed as in Figure 1D. All histograms show mean values from 3 independent experiments; bars indicate SD.

To confirm the role of STAT1 and STAT2 in mediating the effect of IFN- $\alpha$ on HBV replication, we generated a replication-competent HBV genome bearing ISRE-inactivating mutations (IRESmt) (ref. 13 and Figure 4A). As expected, cccDNA molecules harboring an ISREmt do not recruit STAT2 (Figure 4B). We found that ISREmt HBV transcribes less pgRNA (Figure 4C) and accordingly replicates less efficiently (Figure 4D). Importantly, pgRNA transcription from the ISREmt HBV cccDNA is not repressed by IFN- $\alpha$ treatment (Figure 4C), supporting the notion that the ISRE indeed mediates IFN- $\alpha$ transcriptional repression of cccDNA transcription. On the other hand, the observed reduction of pgRNA production in untreated ISREmt HBV-replicating cells (Figure 4C) would suggest that the binding of STATs, and possibly of other transcription factors/coactivators, to the WT HBV ISRE contributes, in the absence of exogenous IFN- $\alpha$, to cccDNA transcription and replication. Furthermore, the replicative activity of the ISREmt HBV displays a reduced but still significant repression by IFN- $\alpha$ (Figure 4D) that likely reflects the contribution of the transcription-independent effects mediated by IFN- $\alpha$ (7).

IFN- $\alpha$ affects the epigenetic control of the HBV cccDNA minichromosome. To gain further mechanistic insights in the epigenetic regulation of the HBV minichromosome function by IFN- $\alpha$ and to investigate how active cccDNA-bound STAT complexes are converted into repressive ones, we performed additional cccDNA ChIP experiments. HBV-transfected HepG2 cells were exposed to IFN- $\alpha$ for 48 hours, and chromatin was immunoprecipitated using antibodies that specifically recognize the histone deacetylases HDAC1 and hSirt1, the polycomb protein enhancer of zeste homolog 2 (Ezh2), and the transcription factors YY1 (yin yang 1). HDAC1 and hSIRT1 were previously shown to bind the cccDNA, and their recruitment and activity correlated with decreased viral replication in cell culture and, in the case of HDAC1, in the liver of HBV chronic hepatitis patients $(3,4)$. YY1 is a ubiquitous zincfinger transcription factor of the Polycomb Group (PcG) protein family that, depending on the binding context, can activate or

repress the transcription of many viral and cellular genes (24). YY1-binding sites have been located upstream of DR1 in the HBV genome (25). To exert its repressive function, YY1 interacts with different HDACs (26) and with Ezh2, a PcG chromatin-modifying enzyme with histone lysine methyltransferase (HKMT) activity (27). As shown in Figure 5A, 48 hours after transfection, when the cccDNA-bound histones $\mathrm{H} 4$ are significantly hypoacetylated in IFN- $\alpha$-treated cells (Figure 1D), the recruitment of HDAC1, Sirt1, Ezh2, and YY1 is increased. It is noteworthy that Ezh2 occupancy on the cccDNA is still conserved 48 hours after IFN- $\alpha$ treatment is stopped (Figure 5B). Accordingly, pgRNA transcription (Figure $5 \mathrm{~B}$ ) and cytoplasmic HBV core particle production (Figure 5B) are both persistently repressed after IFN- $\alpha$ release. These results indicate that IFN- $\alpha$ induces a persistent recruitment of several corepressors and components of the polycomb repressive complex 2 (PRC2) that target histone tail acetylation and methylation, thus providing a molecular mechanism for long-term off-treatment IFN- $\alpha$ repression of HBV transcription (Figure 6).

Finally, we studied the impact of the ISRE mutation on cccDNA chromatin status in response to IFN- $\alpha$. We found that the ISREmt HBV cccDNA does not recruit, as expected, STAT2 proteins and displays, according to its reduced transcription/replication capacity, a reduced acetylation of cccDNA-bound $\mathrm{H} 4$ histones that is not further modified by IFN- $\alpha$ (Figure 5C). We also show that the ISREmt HBV cccDNA fails to recruit HDAC1 in response to IFN- $\alpha$ (Figure 5D), thus further linking the presence of a functional ISRE with the epigenetic changes of the cccDNA chromatin that characterize the transcription repression response to IFN- $\alpha$. Interestingly, the ISREmt cccDNA displays an increased hSirt1 binding in the absence of IFN- $\alpha$ stimulation and further recruits hSirt 1 in response to IFN- $\alpha$ stimulation. These observations suggest that the presence of hSirt 1 on the ISREm cccDNA might contribute to its reduced transcriptional activity in unstimulated cells, whereas its further recruitment after IFN treatment might mediate in part the IFN- $\alpha$ repression of the ISREmt HBV cccDNA.

\section{Discussion}

Together, our results identify a new molecular mechanism by which IFN- $\alpha$ inhibits HBV replication. We show that IFN- $\alpha$ inhibits cccDNA-driven transcription of genomic and subgenomic RNAs, both in HBV-replicating cells in culture and in HBV-infected chimeric uPA/SCID mice repopulated with primary human hepatocytes. By generating a replication-competent HBV genome carrying ISREinactivating mutations, we also linked IFN- $\alpha$ inhibitory activity with the presence of a functional ISRE. ISREmt HBV transcribes less pgRNA, replicates less efficiently, and, more importantly, cannot be repressed by IFN- $\alpha$. Both STAT1 and STAT2 transcription factors and their phosphorylated "active" forms are recruited on the cccDNA, and their binding is reduced by IFN- $\alpha$ treatment. Since, under the same experimental conditions, several cellular ISGs were activated (Supplemental Figure 1), one possible mechanism to explain IFN- $\alpha$-repressive activity on cccDNA transcription might be an IFN- $\alpha$-induced redistribution of active STAT1/ STAT2 complexes from the viral minichromosome to cellular target genes. However, we also found that, in response to IFN- $\alpha$, cccDNA-bound histones become hypoacetylated and both components of the transcriptional repressor complex PRC2, namely YY1 and Ezh2, and the histone deacetylases HDAC1 and hSirt1 are actively recruited on the cccDNA. These latter observations suggest an active mechanism for IFN- $\alpha$ repression of HBV transcription, 
A

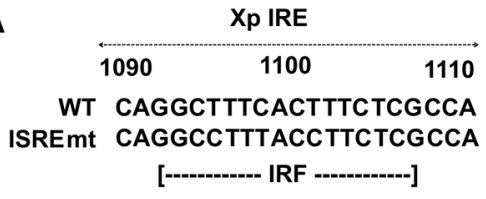

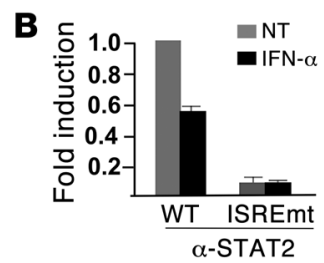

C

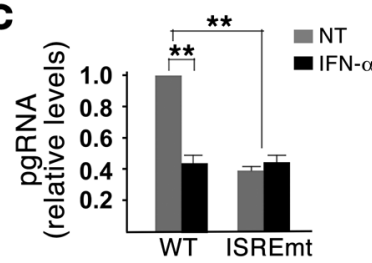

D

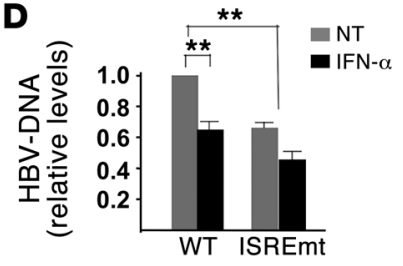

Figure 4

HBV ISRE mediates IFN- $\alpha$ transcriptional repression. (A) Sequence of the HBV enhancer $1 / X$ gene promoter around the HBV ISRE. ISRE mutations are shown. The nucleotide substitutions do not alter the HBV polymerase polypeptide sequence. (B) Chromatin prepared from untreated and IFN- $\alpha$-treated HepG2 cells transfected with WT or ISREmt HBV genomes was immunoprecipitated with a relevant control IgG or specific anti-STAT2 antibodies. Immunoprecipitated chromatin was analyzed by qPCR and results expressed as in Figure 1D. (C) mRNAs were prepared from untreated and IFN- $\alpha$-treated HepG2 cells transfected with WT and ISREmt HBV genomes, and HBV pregenomic RNA was quantified by qPCR using specific primers. GAPDH amplification was used to normalize for equal loading of each RNA sample. (D) Cytoplasmic HBV core particles were isolated from untreated and IFN- $\alpha$-treated HepG2 cells 48 hours after transfection with monomeric linear full-length WT or ISREmt genomes. Results are expressed as in Figure 1A. Results are shown as mean values from 3 independent experiments; bars indicate SD. $P$ values were determined using Student's $t$ test. ${ }^{* *} P<0.01$.

where changes of cccDNA-bound transcriptional regulators translate into changes in the epigenetic control of cccDNA minichromosome function, rather than a simple "passive" relocation of ISFG3 complexes. It will be important in the future to define the precise nature of the signals that IFN- $\alpha$ provides in order to not only modify the composition and the function of STAT1and STAT2-containing complexes, but also the organization of other cccDNA-bound transcription factors and regulators. In this respect, it has been recently reported that inflammatory signaling involving the induction of the TNF- $\alpha$ receptor/p38 MAPK cascade results in Ezh2 phosphorylation, increased binding to YY1, and transcriptional repression of target genes (28).

Virion productivity, being defined as the ratio between the number of intrahepatic relaxed circular HBV DNA and cccDNA molecules, depends on pgRNA synthesis and thus also reflects cccDNA transcription. From a more translational point of view, our data also provide a molecular frame explaining previous studies reporting a very strong HBV suppression, with a $99 \%$ inhibition of intrahepatic virion productivity, in patients who had been treated with the combination of IFN- $\alpha$ and the nucleotide ana$\log$ adefovir dipivoxil for 1 year (29). The observation that during the following 2 years, when patients were shifted to ADV monotherapy, the suppression of viral productivity was reduced to $76 \%$, although the clinical benefit was maintained in most patients, suggests that IFN- $\alpha$ may have direct antiviral effects in vivo that are qualitatively different from those of HBV DNA polymerase inhibitors. Indeed, PEG-IFN- $\alpha$ results in the highest rate of offtreatment sustained virological response after a 1-year course of therapy (5). This sustained virological suppression, achieved in $30 \%-35 \%$ of $\mathrm{HBeAg}$-positive patients and $20 \%-25 \%$ of $\mathrm{HBeAg}$ negative patients, is commonly thought to reflect the transition, induced by IFN- $\alpha$ treatment, to the "immune-control" phase that characterizes the inactive HBsAg carrier state. Our results indicate that IFN- $\alpha$ induces a persistent condition of "active epigenetic control" of HBV cccDNA minichromosome, involving all HBV transcripts, that may contribute to the persistent, yet reversible, "offtherapy" inhibition of HBV replication (Figure 6). Understanding IFN- $\alpha$-induced mechanisms acting both at posttranscriptional $(7,12)$ and transcriptional levels to suppress HBV replication and how these IFN- $\alpha$ direct activities are linked with other signals from both adaptive and innate immune responses may assist in the development of more effective therapeutic approaches.

\section{Methods}

Cell cultures and IFN- $\alpha$ treatments. HepG2 hepatoma cells were maintained in DMEM supplemented with 10\% FBS (Gibco; Invitrogen), 1\% penicillin/streptomycin, and $1 \%$ glutamine (Sigma-Aldrich). IFN- $\alpha$ was used at a final concentration of $1000 \mathrm{IU} / \mathrm{ml}$ and was added directly to the culture medium every 24 hours, starting 4 hours after transfection, unless otherwise indicated.

Transient transfection of full-length HBV DNA genomes. Monomeric linear full-length (14) WT and ISREmt HBV genomes were released from the PCR.HBV.A.EcoRI (4) and the pCR.ISREmt.A.EcoRI plasmids using EcoRI-PvuI (New England Biolabs) The pCR.ISREmt.A.EcoRI has been generated by site-directed mutagenesis (QuikChange Site-Directed Mutagenesis Kit; Stratagene Inc.) from the pCR.HBV.A.EcoRI plasmid. The oligonucleotides used for mutagenesis were as follows: sense, $5^{\prime}$-ATACAAGCTAAACAGGCCTTTACCTTCTCGCCAACTTACAAG-3'; antisense, 5' -CTTGTAAGTTGGGCAGAAGGTAAAGGCCTGTTTAGC-3'. These nucleotide substitutions in the ISRE sequence have been shown to abolish ISGF3 binding in EMSA assays (13), but do not alter the HBV polymerase polypeptide sequence. Linear HBV monomers were transfected into HepG2 human hepatoma cells using the Mirrus Bio transIT-LT1 (Mir 2300A). Briefly, HepG2 cells were seeded at a density of 2-3 million cells in 100-mm-diameter Petri dishes and transfected 24 hours later with $1 \mu \mathrm{g}$ to $2 \mu \mathrm{g}$ of digested HBV DNA. Unless specified otherwise, culture medium was changed 1 day after transfection and cells were harvested at the indicated times. All transfection included $0.5 \mu \mathrm{g}$ of GFP expression vector to assess transfection efficiency (HepG2 cells, range 28\%-32\%). To exclude nonhomologous recombination events at the level of the 2 ends of the transfected linear HBV DNA and the possible generation of circular HBV DNA molecules carrying sequence modifications at the recombination site, the HBV region spanning the predicted ends of the linear dsDNA was amplified and sequenced (3).

Purification and quantification of core particles associated with HBV DNA from HBV-replicating cells. To purify HBV DNA from intracellular core particles, transfected cells were washed once with ice-cold PBS and lysed in $50 \mathrm{mmol}$ Tris-HCl, pH 7.4, 1 mmol EDTA, and 1\% NP-40 (lysis buffer A). Nuclei were pelleted by centrifugation for 1 minute at $10,000 \mathrm{~g}$. The supernatant was adjusted to $100 \mathrm{mmol} \mathrm{MgCl}$ and treated with $100 \mathrm{mg} / \mathrm{ml}$ of DNase I for 30 minutes at $37^{\circ} \mathrm{C}$. The reaction was stopped by adding EDTA to a final concentration of $25 \mathrm{mmol}$. Protein was digested with $0.5 \mathrm{mg} / \mathrm{ml}$ proteinase $\mathrm{K}$ and $1 \%$ SDS for 2 hours at $50^{\circ} \mathrm{C}$. Nucleic acids were purified by phenolchloroform (1:1) extraction and ethanol precipitation adding glycogen and examined by Southern blot analysis (3). HBV DNA was quantified by real-time PCR in a Light Cycler instrument (Roche) using the following 
A

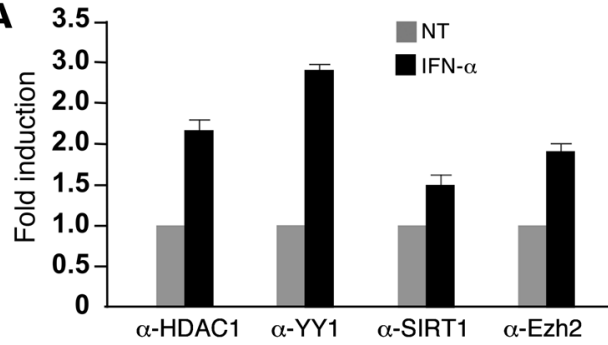

B

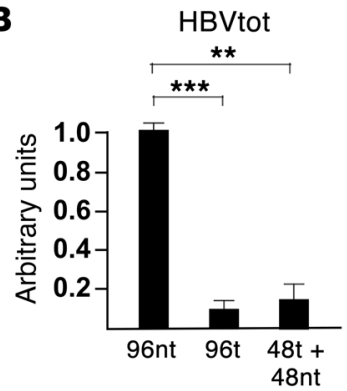

C

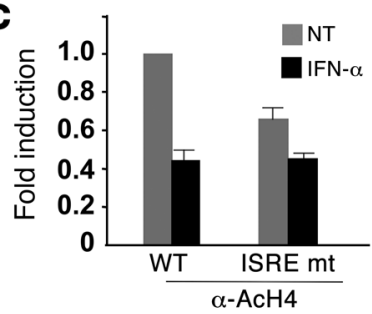

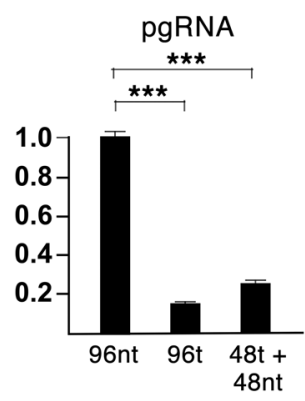
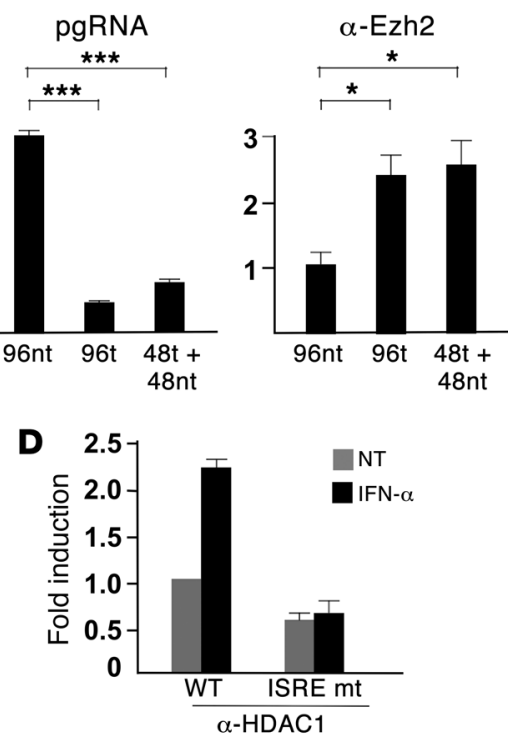

\section{Figure 5}

IFN- $\alpha$ modulates the epigenetic control of cccDNA function by affecting the recruitment of chromatin-modifying enzymes. (A) Cross-linked chromatin from untreated and IFN- $\alpha$-treated HepG2 cells transfected with WT HBV genomes was immunoprecipitated with a relevant control IgG or anti-HDAC1, anti-YY1, anti-hSirt1 and anti-EzH2 antibodies and analyzed as in Figure 1D. (B) HepG2 cells, transfected as in $\mathbf{A}$, were either left untreated (96nt), or treated for 96 hours after transfection with IFN- $\alpha$ (96t), or treated with IFN- $\alpha$ for 48 hours and then left untreated for 48 hours $(48 t+48 n t)$. Left panel: cross-linked chromatin immunoprecipitated with a relevant control IgG or anti-Ezh2 antibody was analyzed as in Figure 1D. pgRNA (middle panel) and cytoplasmic core particles HBV-DNA (right panel) were quantified by real-time qPCR. Results are expressed as in Figure 1. (C and $\mathbf{D})$ Chromatin was prepared from untreated and IFN- $\alpha$-treated HepG2 cells transfected with WT or ISREmt HBV genomes and immunoprecipitated with a relevant control IgG or anti-AcH4 (B) or anti-HDAC1 (C) antibodies. Immunoprecipitated chromatin was analyzed by qPCR and results expressed as in Figure 1D. All histograms show the mean from 3 independent experiments; bars indicate SD. $P$ values were determined using Student's $t$ test. ${ }^{*} P<0.05 ;{ }^{* *} P<0.01 ;{ }^{* *} P<0.001$. primers and probes: forward, 5'-CTCGTGGTGGACTTCTCTC-3', and reverse $5^{\prime}$-CAGCAGGATGAAGAGGAA-3'. We also used specific FRET hybridization probes: 5'-CACTCACCAACCTCCTGTCCTCCAA-FL-3', Red640, 5'-TGTCCTGGTTATCGCTGGATGTGTCT-3'. Amplifications were performed as follows: $95^{\circ} \mathrm{C}$ for 5 minutes, followed by 45 cycles at $95^{\circ} \mathrm{C}$ for 10 seconds, $58^{\circ} \mathrm{C}$ for 10 seconds, and $72^{\circ} \mathrm{C}$ for 20 seconds.

$H B V c c c D N A$ quantification. HepG 2 cells were collected at the indicated times after transfection, resuspended in lysis buffer $\mathrm{A}$ (see above), and incubated 10 minutes at $4{ }^{\circ} \mathrm{C}$. Lysates were centrifuged 1 minute at 10,000 g; pelleted nuclei were resuspended in lysis buffer B (10 mM Tris-HCL, $10 \mathrm{mM}$ EDTA, $150 \mathrm{mM} \mathrm{NaCl}, 0.5 \% \mathrm{SDS}$, and $0.5 \mathrm{mg} / \mathrm{ml}$ protein $\mathrm{K}$ ) and incubated overnight at $37^{\circ} \mathrm{C}$. Nucleic acids were purified by phenol-chloroform (1:1) extraction and ethanol precipitation. $500 \mathrm{ng}$ aliquots of each extracted DNA were treated for 45 minutes at $37^{\circ} \mathrm{C}$ with $10 \mathrm{U}$ plasmid safe DNase I (Epicentre Inc.). DNase was inactivated by incubating the reactions for 30 minutes at $70^{\circ} \mathrm{C}$. Real-time PCR experiments were performed in a Light-Cycler (Roche Diagnostics) using a 201 reaction volume containing $20 \mathrm{ng}$ of DNA, $3 \mathrm{mmol} / \mathrm{MgCl} 2,0.5 \mathrm{mmol} / \mathrm{l}$ of forward and reverse primers, $0.2 \mathrm{mmol} / 1$ of 3 '-fluorescein-labeled (FL-labeled) probe, and $0.4 \mathrm{mmol} / \mathrm{l}$ of $5^{\prime}$-Red640-labeled (R640-labeled) probe. Forward and reverse primers were as follows: $5^{\prime}$-CTCCCCGTCTGTGCCTTCT- $3^{\prime}$ (NCCC1 nt 1548-1566) and 5'-GCCCCAAAGCCACCCAAG-3' (CCCAS2 nt 1903-1886), respectively. Hybridization probes were 5'-GTTCACGGTGGTCTCCATGCAACGT-FL-3' and 5'-R640-AGGTGAAGCGAAGTGCACACGGACC-3', respectively. Amplification was performed as follows: $95^{\circ} \mathrm{C}$ for 10 minutes, then 45 cycles of $95^{\circ} \mathrm{C}$ for 10 seconds, $62^{\circ} \mathrm{C}$ for 10 seconds, and $72^{\circ} \mathrm{C}$ for 20 seconds. The efficacy of DNase treatment in the elimination of OC and SS forms of HBV DNA prior to
PCR was confirmed by the abrogation of the PCR amplification of HBV DNA extracted from cytoplasmic viral particles by the nonselective HBV oligonucleotide primers targeting the HBs ORF (3). Globin amplification was performed using the Light-Cycler Globin Control Kit (Roche Diagnostics). Serial dilutions of a plasmid containing a monomeric genotype A HBV insert (Clonit Srl.) were used as quantification standards. Southern blot analysis was performed following standard procedures as previously described (3).

$H B V$ RNAs and cellular $m R N A$ analysis. Total RNA was extracted from HepG 2 cells 48 hours and 96 hours after transfection with $1 \mathrm{mg}$ of linear monomeric HBV DNA using the TRIzOL reagent (Invitrogen) as recommended by the manufacturer. The RNA samples were treated with RQ1 RNase-Free DNase (Promega) for 30 minutes at $37^{\circ} \mathrm{C}$ and stored until used. RNA quality and quantity were monitored by ethidium bromide staining and by UV absorbance. For pgRNA analysis, $2 \mathrm{mg}$ of DNase-treated RNA was reverse transcribed and amplified by the ThermoScript RT-PCR System (Invitrogen). Then $2 \mu \mathrm{l}$ of each cDNA was quantified by real-time PCR analysis (Light Cycler; Roche Diagnostics) using the following pgRNAspecific primers and probes: forward primer, 5 '-GCCTTAGAGTCTCCTGAGCA-3' ${ }^{\prime}$, reverse primer, 5'-GAGGGAGTTCTTCTTCTAGG-3', FRET hybridization probes, 5'-AGTGTGGATTCGCACTCCTCCAGC-FL-3', and Red640-5'ATAGACCACCAAATGCCCCTATCTTATCAAC-3'. The h-GAPDH housekeeping gene Light Cycler Set (Roche Diagnostics) was used to normalize the RNA samples.

For Northern blot analysis, $25 \mu \mathrm{g}$ of total RNA per sample was separated on a $1 \%$ formaldehyde-agarose gel and blotted onto Zeta-Probe GT membranes (Bio-Rad Laboratories). Radioactive probes were prepared by random priming protocol, using either full-length HBV DNA or 18S cDNA 


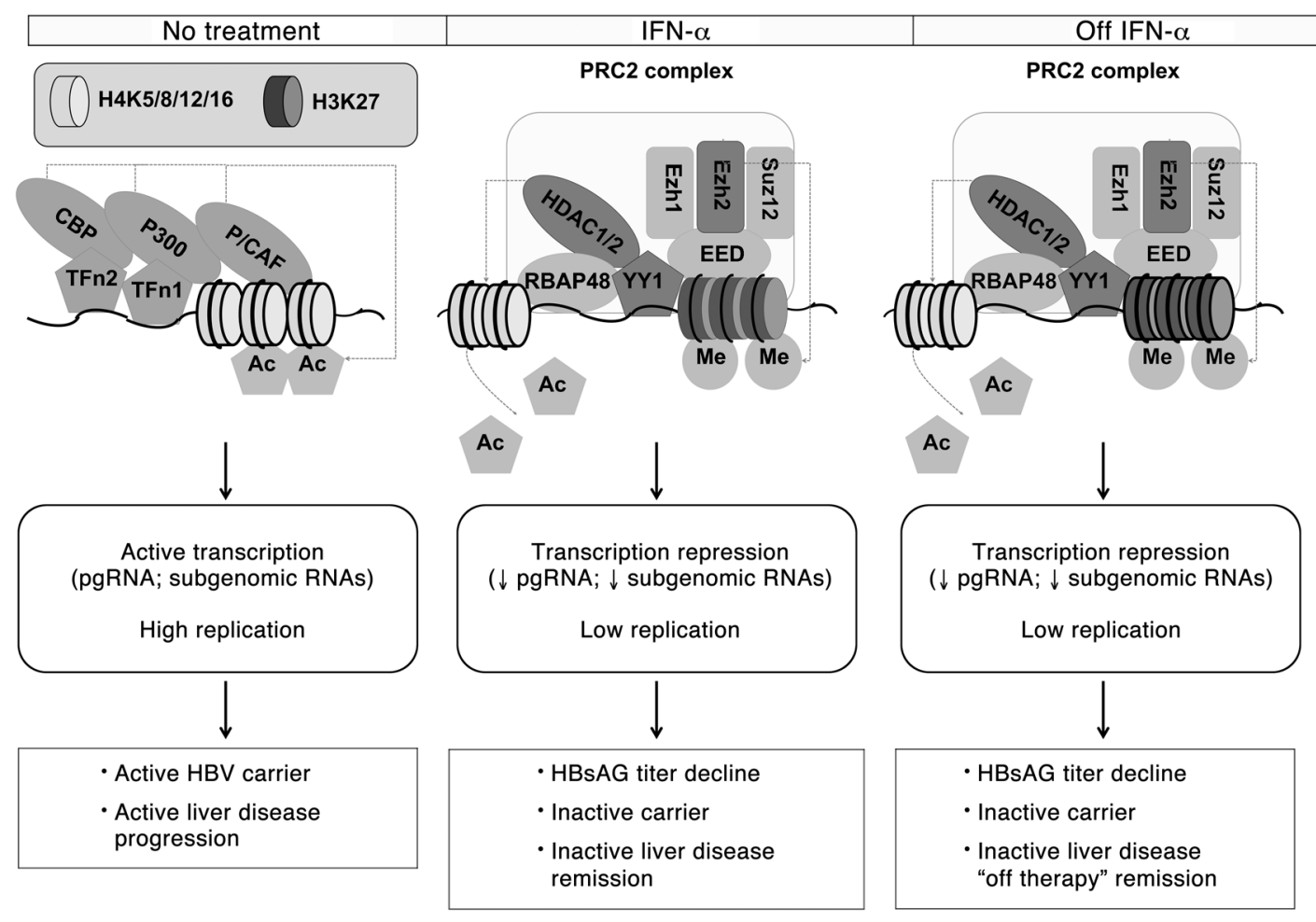

\section{Figure 6}

Schematic representation of cccDNA chromatin changes in response to IFN- $\alpha$ treatment. cccDNA-bound histone acetylation status and the recruitment of chromatin-modifying enzymes onto the viral minichromosome change in relation to viral replication and IFN- $\alpha$ treatment. The drawings reflect what is observed in an in vitro replication system. The translation into the clinical scenario as it is observed in patients (lower boxes) is inferred, and it awaits to be confirmed by ex vivo experiments. In the context of high HBV replication and in the absence of IFN- $\alpha$ treatment, cccDNA-bound histones are hyperacetylated, cccDNA-associated chromatin is in an open configuration, pgRNA is actively transcribed, and HBV replication is unrestricted (left drawing). The clinical correlate is an active HBV carrier with high HBV viremia, reflecting high levels of intrahepatic viral replication and liver disease progression. In response to IFN- $\alpha$, HDACs (HDAC1 and Sirt1) substitute HAT enzymes (p300, CBP, and P/CAF) on the cccDNA, and a PRC2-repressor complex is recruited. This leads to histone deacetylation/methylation at specific lysine residues, a "closed" chromatin configuration, and a striking reduction of pgRNA transcription, HBsAg synthesis, and HBV replication (middle drawing). In the clinical setting, this would translate into a rapid serum HBsAg decline and viral suppression (inactive carrier) with disease remission. When treatment is stopped, the chromatin changes imposed by IFN- $\alpha$ tend to persist (right drawing) resulting in the "off-therapy" maintenance of the virological suppression and clinical improvement (achieved in 30\%-35\% of HBeAg-positive patients and $20 \%-25 \%$ of HBeAg-negative patients). Darker forms in the middle and right drawings indicate components of the PRC2 complex whose recruitment has been directly investigated in this paper.

templates and ${ }^{32} \mathrm{P} \alpha$-dCTP (Amersham). After hybridization, the membrane was washed and exposed to X-Omat film (Kodak Inc.) at $-80^{\circ} \mathrm{C}$.

For the analysis of cellular ISG expression by TaqMan Low-Density Arrays (TLDAs) (Applied Biosystems), total RNAs were reverse transcribed using the Random Primers Superscript Kit (Invitrogen), and 200 ng of each complementary DNA was loaded in double on a 95-ISG customized TLDA with 18S RNA used as a control. TLDAs were run on an AB 7900HT thermocycler, and real-time PCR data were collected and analyzed using the SDS 2.2 program (Applied Biosystems).

Generation of human chimeric mice, HBV infection experiments, and IFN treatment. Alb uPA transgenic mice (Jackson Laboratories) crossed with SCID/beige mice (Taconic Farms) were housed and maintained under specific pathogen-free conditions in accordance with institutional guidelines under approved protocols. The presence of the uPA transgene and the maintenance of the SCID phenotype were determined as reported (15). Three- to four-week-old homozygous uPA SCID/beige mice were anesthetized with isoflurane and injected intrasplenically with $1 \times 10^{6}$ cryopreserved and thawed viable human hepatocytes (30) isolated from 1 liver specimen obtained from a reduced size liver transplant. Informed consent was obtained from donors, and all procedures were approved by the ethical committee of the city and state of Hamburg according to the principles of the Declaration of Helsinki. Human hepatocyte repopulation levels were determined by measuring HSA concentrations in mouse serum with the Human Albumin ELISA Quantitation Kit (Bethyl Laboratories, Biomol $\mathrm{GmbH}$ ) as recommended by manufacturers. Human chimeric animals displaying HSA concentrations of 1 or more $\mathrm{mg} / \mathrm{ml}$ were used for the study. Ten to twelve weeks after mice received a single peritoneal injection of HBV-positive mouse-derived serum $\left(2 \times 10^{7} \mathrm{HBV}\right.$ DNA copies), stably infected animals were treated with IFN- $\alpha$ (1300 IU/g body weight, daily, in $200 \mu \mathrm{l}$ ), while control animals received saline. Liver specimens removed at sacrifice were snap-frozen in liquid nitrogen for further histological and molecular analyses. All animal experiments were conducted in accordance with the European Communities Council Directive (86/EEC), and were approved by the city of Hamburg, Germany.

Virological measurements in UPA chimeric mice. After homogenization of the mouse liver specimens, DNA and RNA were extracted in parallel using the Master Pure DNA Purification Kit (Epicentre; Biozym) and RNeasy RNA Purification Kit (QIAGEN). Human hepatocyte 
genome equivalents and intrahepatic viral loads were determined per ng genomic DNA isolated from chimeric livers. After treating purified DNA with $20 \mathrm{U}$ plasmid-safe DNase I (Epicentre; Biozym) to enrich the cccDNA fraction, intrahepatic cccDNA amounts were determined using cccDNA-specific primers and FRET probes as reported above. Cloned HBV DNA was used to establish a standard curve for quantification. cccDNA copies were normalized for human hepatocyte contents using the $\beta$-globin Quantification Kit (Roche Applied Science) $(15,29)$. Viral RNA was reverse transcribed using oligo-dT primers and the Transcriptor Kit (Roche Applied Science) and quantified by using primers specific for pgRNA and total HBV-RNA (tot) sequences. Steady-state levels of intrahepatic preS/S RNAs were determined by subtracting pgRNA amounts from total HBV RNAs (pgRNA + preS/S RNA) estimated in the same RNA preparation as reported (31), and values were normalized using human-specific GAPDH primers (QuantiTect Primer Assay; QIAGEN) (15). Northern blot analysis was performed as described above.

ChIP assays. 48 hours after transfection with linear HBV monomers, HepG2 cells were resuspended in $1 \mathrm{ml}$ of ChIP lysis buffer $(50 \mathrm{mM}$ Tris HCL, $\mathrm{pH} 8,0.5 \% \mathrm{NP} 40,1 \mathrm{mM}$ EDTA, and $100 \mathrm{mM} \mathrm{NaCL}$ ) and incubated 10 minutes at $4{ }^{\circ} \mathrm{C}$. The lysate was centrifuged at $10,000 \mathrm{~g}$ for 2 minutes to pellet the nuclei. The supernatant was removed, and the nuclei were fixed in $1 \%$ formaldehyde for 30 minutes at $4^{\circ} \mathrm{C}$. Isolated cross-linked nuclei were extracted with a $20 \mathrm{mM}$ Tris, $\mathrm{pH} 8,3$ $\mathrm{mM} \mathrm{MgCl}_{2}, 20 \mathrm{mM} \mathrm{KCl}$ buffer containing protease inhibitors, pelleted by microcentrifugation, and lysed by incubation in SDS lysis buffer ( $1 \%$ sodium dodecyl sulfate, $10 \mathrm{mM}$ EDTA, $50 \mathrm{mM}$ Tris-chloride, $\mathrm{pH}$ 8.1) containing protease inhibitors. The resulting chromatin solution was sonicated for 5 pulses of 45 seconds at $80 \%$ power to generate 300 to 1000-bp DNA fragments using a Bioruptor Sonicator (Diagenode Inc). After microcentrifugation, the supernatant was diluted 1:10 in a dilution buffer $(0.01 \%$ sodium dodecyl sulfate, $1.1 \%$ Triton X-100, $1.2 \mathrm{mM}$ EDTA, $16.7 \mathrm{mM}$ Tris-chloride, $\mathrm{pH}$ 8.1, $167 \mathrm{mM} \mathrm{NaCl}$, containing protease inhibitors), precleared with blocked Protein G Plus (Pierce), and divided into aliquots. The chromatin was then subjected to immunoprecipitation for $14-16$ hours at $4{ }^{\circ} \mathrm{C}$ using antibodies specific to H4 (ab1791; Abcam), AcH4 (06-866, Upstate; rabbit polyclonal IgG recognizing histone $\mathrm{H} 4$, which is tetra-acetylated at lysines 6, 9, 13, and 17), Sirt1 (sc15404/; Santa Cruz Biotechnology Inc.), HDAC1 (no. 06-720; Upstate), STAT1 (sc476; Santa Cruz Biotechnology Inc.), STAT2 (sc476; Santa Cruz Biotechnology Inc.), phospho-STAT1 (9171; Cell Signaling), phospho-STAT2 (07224; Upstate), YY1 (sc1703; Santa Cruz Biotechnology Inc.), and EZH2 (3147; Cell Signaling). Immunoprecipitations with nonspecific immunoglobulins (Santa Cruz Biotechnology Inc.) were included in each experiment as a negative control. After the reverse cross-linking, immunoprecipitated chromatin was purified by phenol/chloroform (1:1) extraction and ethanol precipitation and analyzed by either PCR amplification using specific primers for the HBV
cccDNA (NCC1 and CCCAS) or real-time PCR using the same cccDNA primers and specific probes (FL and Red).

ChIP assays of liver specimens of chronically HBV-infected uPA chimeric mice were performed with the following modifications: liver tissues were homogenized in $500 \mu \mathrm{l}$ lysis buffer $(5 \mathrm{mM}$ PIPES, $85 \mathrm{mM} \mathrm{KCl}$, $0.5 \% \mathrm{NP} 40$ ) and incubated at $4^{\circ} \mathrm{C}$ for 10 minutes. After microcentrifugation, the pelleted nuclei were fixed in $1 \%$ formaldehyde for $15 \mathrm{~min}$ utes at $4^{\circ} \mathrm{C}$. After sonication and dilution, the chromatin was subjected to immunoprecipitation for $14-16$ hours at $4^{\circ} \mathrm{C}$ using antibodies specific to acetylated H4 (06-866; Upstate) or nonspecific immunoglobulins (BD Biosciences). Immunoprecipitated DNA was extracted using the Master Pure DNA Purification Kit (Epicentre; Biozym). After treatment with 20 U plasmid-safe DNAase I (Epicentre; Biozym), DNA was analyzed by real-time PCR using cccDNA-specific primers and FRET probes as reported above.

Immunoblotting. Cells were lysed in RIPA buffer (50 mM Tris, $\mathrm{pH}$ 7.6, $1 \% \mathrm{NP}-40,140 \mathrm{mM} \mathrm{NaCl}, 0.1 \% \mathrm{SDS}$ ), and the insoluble pellet was discarded after centrifugation. Protein concentration was determined by the BCA protein assay reagent (Bio-Rad). Protein lysates were transferred to a nitrocellulose membrane and incubated with STAT1 (sc346; Santa Cruz Biotechnology Inc.) and STAT2 (sc476; Santa Cruz Biotechnology Inc.) antibodies.

Statistics. $P$ values were determined using the 2 -tailed Student's $t$ test. The Wilcoxon rank-sum test was used for nonparametric pair-wise comparisons. $P<0.05$ was considered significant.

\section{Acknowledgments}

M. Levrero and G. Raimondo are supported by grants from Associazione Italiana per la Ricerca sul Cancro and PRIN-MIUR (Progetti di Ricerca di Interesse Nazionale del Ministero dell'Istruzione, dell'Università e della Ricerca). M. Levrero and J. Petersen were also supported by the Vigilance Network for the management of antiviral drug resistance (Virgil). M. Dandri is supported by the Deutsche Forschungsgemeinschaft (SFB 481) and receives research support from Roche Palo Alto LLC (grant number 09-631).

Received for publication May 3, 2011, and accepted in revised form November 9, 2011.

Address correspondence to: Maura Dandri, Department of Internal Medicine, University Medical Hospital Hamburg-Eppendorf, Martinistrasse 52, 20246 Hamburg, Germany. Phone: 49.40.741052949; Fax: 49.40.741057232; E-mail: m.dandri@uke.de. Or to: Massimo Levrero, Department of Internal Medicine, DMISM, Laboratory of Gene Expression, Sapienza University, Viale del Policlinico 155, 00161 Rome, Italy. Phone: 39.06.49970892; Fax: 39.06.4452388; E-mail: massimo.levrero@uniroma1.it.
1. Nassal M. Hepatitis B viruses: reverse transcription a different way. Virus Res. 2008;134(1-2):235-249.

2. Levrero M, Pollicino T, Petersen J, Belloni L, Raimondo G, Dandri M. Control of cccDNA function in hepatitis B virus infection. $J$ Hepatol. 2009;51(3):581-592.

3. Pollicino T, et al. Hepatitis B virus replication is regulated by the acetylation status of hepatitis $B$ virus cccDNA-bound $\mathrm{H} 3$ and $\mathrm{H} 4$ histones. Gastroenterology. 2006;130(3):823-837.

4. Belloni L, et al. Nuclear HBx binds the HBV minichromosome and modifies the epigenetic regulation of cccDNA function. Proc Natl Acad Sci U S A. 2009;106(47):19975-19979.

5. European Association For The Study Of The Liver. EASL Clinical Practice Guidelines: management of chronic hepatitis B. J Hepatol. 2009;50(2):227-242. 6. Sadler AJ, Williams BR. Interferon-inducible antiviral effectors. Nat Rev Immunol. 2008;8(7):559-568.

7. Wieland SF, Guidotti LG, Chisari FV. Intrahepatic induction of alpha/beta interferon eliminates viral RNA-containing capsids in hepatitis B virus transgenic mice. J Virol. 2000;74(9):4165-4173.

8 . Xu C, et al. Interferons accelerate decay of replication-competent nucleocapsids of hepatitis B virus. J Virol. 2010;84(18):9332-9340.

9. Li J et al. Inhibition of hepatitis B virus replication by MyD88 involves accelerated degradation of pregenomic RNA and nuclear retention of pre-S/S RNAs. J Virol. 2010;84(13):6387-6399.

10. Tur-Kaspa R, et al. Alpha interferon suppresses hepatitis $\mathrm{B}$ virus enhancer activity and reduces viral gene transcription. J Virol. 1990;64(4):1821-1824.

11. Rang A, Gunther S, Will H. Effect of interferon alpha on hepatitis $B$ virus replication and gene expression in transiently transfected human hepatoma cells. J Hepatol. 1999;31(5):791-799.

12. Uprichard SL, Wieland SF, Althage A, Chisari FV. Transcriptional and posttranscriptional control of hepatitis B virus gene expression. Proc Natl Acad Sci US A. 2003;100(3):1310-1315.

13. Alcantara FF, Tang H, McLachlan A. Functional characterization of the interferon regulatory element in the enhancer 1 region of the hepatitis B virus genome. Nucleic Acids Res. 2002;30(9):2068-2075.

14. Gunther S, Li BC, Miska S, Kruger DH, Meisel H, Will $\mathrm{H}$. A novel method for efficient amplification of whole hepatitis B virus genomes permits 
rapid functional analysis and reveals deletion mutants in immunosuppressed patients. J Virol. 1995;69(9):5437-5444.

15. Petersen J, et al. Prevention of hepatitis B virus infection in vivo by entry inhibitors derived from the large envelope protein. Nat Biotechnol. 2008;26(3):335-341.

16. Lutgehetmann $M$, et al. Hepatitis B virus limits response of human hepatocytes to Interferonalpha in chimeric mice. Gastroenterology. 2011; 140(7):2074-2083.

17. Caselmann W H, Meyer M, Scholz S, Hofschneider $\mathrm{PH}$, Koshy R. Type I interferons inhibit hepatitis $B$ virus replication and induce hepatocellular gene expression in cultured liver cells. J Infect Dis. 1992;166(5):966-971.

18. Hayashi Y, Koike K. Interferon inhibits hepatitis $B$ virus replication in a stable expression system of transfected viral DNA. J Virol. 1989;63(7):2936-2940.

19. Guo JT, Pryce M, Wang X, Barrasa MI, Hu J, Seeger C. Conditional replication of duck hepatitis B virus in hepatoma cells. J Virol. 2003;77(3):1885-1893.

20. Hiraga $\mathrm{N}$, et al. Absence of viral interference and different susceptibility to interferon between hepatitis
$B$ virus and hepatitis $C$ virus in human hepatocyte chimeric mice. J Hepatol. 2009;51(6):1046-1054.

21. Lucifora Lucifora J, et al. Hepatitis B Virus X protein is essential to initiate and maintain virus replication after infection. J Hepatol. 2011;55(5):996-1003.

22. Robertson G, et al. Genome-wide profiles of STAT1 DNA association using chromatin immunoprecipitation and massively parallel sequencing. Nat Methods. 2007;4(8):651-657.

23. Testoni B, Völlenkle C, Guerrieri F, Gerbal-Chaloin S, Blandino G, Levrero M. Chromatin dynamics of genes activation and repression in response to IFNalpha reveal new roles for STAT2 and phosphoSTAT2. J Biol Chem. 2011;286(23):20217-20227.

24. Gordon S, Akopyan G, Garban H, Bonavida B. Transcription factor YY1: structure, function, and therapeutic implications in cancer biology. Oncogene. 2006;25(8):1125-1142.

25. Nakanishi-Matsui M, Hayashi Y, Kitamura Y, Koike K. Integrate hepatitis B virus DNA preserves the binding sequence of transcription factor Yin and Yang 1 at the virus-cell junction. J Virol. 2000;74(12):5562-5568

26. Sankar N, Baluchamy S, Kadeppagari R-K, Sing- hal G, Weitzman S, Thimmapaya B. p300 provides a corepressor function by cooperating with YY1 and HDAC3 to repress c-Myc. Oncogene. 2008;27(43):5717-5728.

27. Caretti G, Di Padova M, Micales B, Lyons GE, Sartorelli V. The Polycomb Ezh2 methyltransferase regulates muscle gene expression and skeletal muscle differentiation. Genes Dev. 2007;18(21):2627-2638.

28. Palacios D, et al. TNF/p38alpha/polycomb signaling to Pax7 locus in satellite cells links inflammation to the epigenetic control of muscle regeneration. Cell Stem Cell. 2010;7(4):455-469.

29. Lutgehetmann $M$, et al. Sequential combination therapy leads to biochemical and histological improvement despite low ongoing intrahepatic hepatitis B virus replication. Antivir Ther. 2008;13(1):57-66.

30. Dandri M, Murray JM, Lutgehetmann M, Volz T, Lohse AW, Petersen J. Virion half-life in chronic hepatitis B infection is strongly correlated with levels of viremia. Hepatology. 2008;48(4):1079-1086.

31. Volz T, et al. Impaired intrahepatic hepatitis B virus productivity contributes to low viremia in most HBeAg-negative patients. Gastroenterology. 2007;133(3):843-852. 